Europhys. Lett., 66 (4), pp. 499-505 (2004)

DOI: $10.1209 / \mathrm{epl} / \mathrm{i} 2003-10233-9$

\title{
Outage probability for soliton transmission
}

\author{
V. Chernyak ${ }^{1}$, M. Chertkov ${ }^{2}$, I. Kolokolov ${ }^{3,2}$ and A. Peleg ${ }^{2}$ \\ 1 Corning Inc., SP-DV-02-8 - Corning, NY 14831, USA \\ 2 Theoretical Division, LANL - Los Alamos, NM 87545, USA \\ 3 Landau Institute for Theoretical Physics - Kosygina 2, 117334 Moscow, Russia
}

(received 31 October 2003; accepted 8 March 2004)

PACS. 42.81.Gs - Birefringence, polarization.

PACS. 78.55.Qr - Amorphous materials; glasses and other disordered solids.

PACS. 05.40.-a - Fluctuation phenomena, random processes, noise, and Brownian motion.

\begin{abstract}
We study the interplay between amplifier noise and birefringent disorder in the case of strongly nonlinear (soliton) type of transmission in optical fibers. Assuming both noise and disorder to be weak, we evaluate the probability distribution function (PDF) of the Bit-Error-Rate (BER) for the values of BER that are much larger than the typical (average) value. The PDF tail that describes probability of the system outage shows log-normal shape, strongly dependent on the fiber length. We also discuss a simple timing shift technique capable of the outage compensation.
\end{abstract}

Nonlinear information transmission in optical fibers when elementary bits are represented by optical solitons constitutes a promising technology that has been a subject of intensive research over the past decades $[1,2]$. In ideal fibers, the information carried by the solitons would be transmitted without any loss. In practice, however, various impairments lead to information loss. Amplifier noise and birefringent disorder represent the two major impairments in both linear and nonlinear transmission regimes. The noise generated by spontaneous emission in optical amplifiers is, therefore, short-correlated both in space and time. Birefringence that stems from variations of the optical fiber core degree of ellipticity is sensitive to external stresses and temperature changes, which leads to its substantial changes along the fiber line. Birefringence is practically frozen, i.e. the typical time scale of the birefringence variations is long compared to the time it takes for a pulse to pass the entire fiber line [3]. Therefore, birefringence can be treated as time-independent disorder, short-correlated in space. The optical communication system performance is usually measured by Bit-Error-Rate (BER) that represents the probability of an incorrect bit identification at the system output. However, because of the quasi-static nature of the birefringent disorder, the system performance may not be characterized in terms of a single number, e.g. some BER averaged over disorder realizations. BER should be rather considered as a number, dependent on a given realization of birefringent disorder, whereas the system performance should be characterized in terms of the Probability Distribution Function (PDF) of BER, with the statistics being collected by averaging over a large number of birefringent-disorder realizations. Note that the co-existence of two very different randomness sources constitutes a common feature of many problems in

(c) EDP Sciences 
the general field of statistical physics of disordered systems, whose behavior is governed by a complex interplay between the short-correlated thermal noise and frozen structural disorder. Thus, in the spin glass theory the object, similar to the PDF of BER, which provides an adequate description of the glassy system is represented by the so-called functional order parameter [4].

Recently, a new approach that describes the joint effect of amplifier noise and birefringent disorder has been developed and applied to the case of linear transmission [5]. It has been shown that the PDF of BER has an algebraic or algebraic-like extended tail. It has been also found [5] that various compensation tricks allow for partial suppression of the long tail. In this manuscript we show that, similarly to the linear case, the PDF of BER for the soliton regime has an extended log-normal tail; however, the parametric dependence of this tail on the fiber length is stronger compared to the bare (no-compensation) linear case. We also note that the problem under consideration is of special interest in terms of theory of nonlinear disordered systems, since we have been able to derive analytical results for the PDF of BER using neither replica calculations nor "cavity" equation approaches [4]. The letter is organized as follows. We first introduce and discuss the equation describing soliton propagation in an optical fiber in the presence of amplifier noise and birefringent disorder. We further evaluate the BER produced by the amplifier noise for a given realization of birefringence disorder. Finally, the $\mathrm{PDF}$ of BER is computed. We conclude the letter with a brief discussion of how the results are modified if the timing jitter ("setting the clock") compensation technique is applied.

The envelope of the electromagnetic field propagating along an optical fiber in the nonlinear regime and in the presence of amplifier noise and birefringence disorder satisfies the following equation $[1,6,7]$ :

$$
\left(\partial_{z}+\hat{m}(z) \partial_{t}-i \partial_{t}^{2}-2 i \sum_{i=1,2}\left|\Psi^{(i)}\right|^{2}\right) \boldsymbol{\Psi}=\boldsymbol{\xi},
$$

represented in dimensionless units. In eq. (1), $z, t$, and $\boldsymbol{\xi}$ are the position along the fiber, time measured in the reference frame traveling together with the soliton, and the amplifier noise, respectively. The envelope $\boldsymbol{\Psi}$ is a two-component field, where the components stand for two polarization states of the optical signal. Birefringent disorder is characterized by a $2 \times 2$ random Hermitian traceless matrix $\hat{m}$. (A simple role played by the term that represents the trace of $\hat{m}$ will be discussed later in the text.) We assume that the terms $\boldsymbol{\xi}$ and $\hat{m}(z) \partial_{t} \boldsymbol{\Psi}$ are both small in comparison with all the other terms in eq. (1), since otherwise the fiber line may not be used for reliable information transmission. The form of the nonlinear term appearing on the righthand side of eq. (1) corresponds to the so-called Manakov case [8], describing the evolution of the electrical field envelop at length scales longer than a characteristic length $z_{\mathrm{c}}$, defined as a typical length scale of changes in the optical polarization [9]. Note, however, that the major results on the $\mathrm{PDF}$ of $\mathrm{BER}$, presented in the letter, are generic, i.e., they are not restricted to the Manakov type of nonlinearity, which is chosen here primarily for the sake of being specific. Equation (1) constitutes a coarse-grain description of propagation where attenuation is assumed to be fully compensated by amplification and the noise source to be distributed homogeneously along the fiber. Such a description is adequate since the transmission system length constitutes several thousand kilometers, whereas the distance between the amplifiers is set approximately to $50-100 \mathrm{~km}$ to compensate the losses. The additive noise $\boldsymbol{\xi}$ generated in optical amplifiers is zero in average and has Gaussian statistics [10] with the correlation time much shorter than the pulse width. Therefore, the statistics of $\boldsymbol{\xi}$ is fully determined by its two-point correlation function

$$
\left\langle\boldsymbol{\xi}^{(i)}\left(z_{1}, t_{1}\right) \boldsymbol{\xi}^{(j) *}\left(z_{2}, t_{2}\right)\right\rangle=D_{\xi} \delta_{i j} \delta\left(z_{1}-z_{2}\right) \delta\left(t_{1}-t_{2}\right),
$$


where the coefficient $D_{\xi}$ characterizes the noise strength determined by the amplifier noise figure [10]. Averaging over birefringent disorder is of a different nature. The birefringence matrix, $\hat{m}$, varies on time scales much longer than the pulse propagation time but shorter than the overall system operation time. Therefore, the disorder statistics is collected over different birefringence states of the same fiber at different times. Since $\hat{m}$ is a Hermitian and traceless matrix it can be expanded in the Pauli matrices $\hat{m}(z)=h_{j}(z) \hat{\sigma}_{j}$ with $h_{j}$ being a real three-component field. This field is zero in average and short-correlated in $z$, since the typical length scale of birefringence variations is small compared to the propagation distance $Z$. As we will see, $h_{j}$ enters all the observables in an integral form and, according to the central-limit theorem (see, e.g., [11]), can be treated as a Gaussian random field described by the following two-point correlation function:

$$
\left\langle h_{i}\left(z_{1}\right) h_{j}\left(z_{2}\right)\right\rangle=D_{m} \delta_{i j} \delta\left(z_{1}-z_{2}\right)
$$

where $D_{m}$ characterizes the disorder strength.

In the ideal case, when noise and disorder are neglected, eq. (1) is integrable [12]. If disorder and noise are weak, eq. (1) can be analyzed in terms of the adiabatic (secular) perturbation theory, see, e.g., $[13,14]$. With a single ideal soliton as an initial $(z=0)$ condition, one seeks for a solution of eq. (1) in the following general form:

$$
\left(\begin{array}{l}
\Psi^{(1)} \\
\Psi^{(2)}
\end{array}\right)=\exp \left[i \varphi+i \beta t+i \hat{\sigma}_{1} \nu\right] \exp \left[-i \hat{\sigma}_{2} \mu\right]\left(\begin{array}{c}
\eta \cosh ^{-1}[\eta(t-y)]+v_{1} \\
v_{2}
\end{array}\right)
$$

where $z$-dependent parameters $\eta, \beta, y, \varphi, \nu, \mu$ (originating from six localized modes of the ideal, $\hat{m}=0, \boldsymbol{\xi}=0$, problem) are modes slowly changing in $z$, whereas $v_{1,2}$ stand for the two components of the continuous-spectrum radiation emitted by the soliton. Initial conditions at $z=0$ read $v_{1,2}(t ; 0)=0, \eta(0)=1, \beta(0)=y(0)=\varphi(0)=\mu(0)=\nu(0)=0$. It can be shown that the results presented in this letter do not depend on this specific choice of the initial conditions for the parameters $\beta, y, \varphi, \nu$, and $\mu$. Obviously, eq. (4) describes a single soliton; the issue of multi-soliton interactions, e.g. of the type considered in [14], will not be addressed in this letter.

Detection of a pulse at the system output that corresponds to $z=Z$ requires a measurement of the pulse intensity $I$,

$$
I=\int \mathrm{d} t G(t)|\Psi(Z, t)|^{2}
$$

where the function $G(t)$ is a convolution of the electrical (current) filter function with the sampling window function that is limiting the information slot. Ideally, $I$ takes a distinct value if the bit encodes " 1 " and is negligible if the bit encodes " 0 ". Both noise and disorder force $I$ to deviate from its ideal value. One declares that the output signal encodes $0 / 1$ if the value of $I$ is smaller/larger than the decision threshold $I_{\mathrm{d}}$. The information is lost if the output value of the bit differs from the input one. The probability of such event should be small in a well-performing fiber, which means that both impairments typically cause only small distortions to a pulse. As we will see below, this statement corresponds to the following two conditions: $D_{\xi} Z^{3} \ll 1, D_{m} Z \ll 1$.

In general, one should consider two contributions to BER, one coming from the case where a "1" bit evolves into "0" $\left(B_{1 \rightarrow 0}\right)$, the other coming from the case where a " 0 " bit becomes "1" $\left(B_{0 \rightarrow 1}\right)$. However, as follows from the forthcoming analysis and ref. [15], for a long system, $Z \gg 1$, the latter contribution is negligible compared to the former one. We therefore focus 
on the situation where the initial value of the bit is " 1 ". The probability of losing a unitary bit for a given disorder realization $\{\hat{m}\}$ is

$$
B\{\hat{m}\}=B_{1 \rightarrow 0}\{\hat{m}\}=\int_{0}^{I_{\mathrm{d}}} \mathrm{d} I P(I \mid\{\hat{m}\}) .
$$

Here $P(I \mid\{\hat{m}\})$ is the probability to detect the output signal of intensity $I$, given that the initial pulse is represented by a single ideal soliton and the birefringent-disorder profile is fixed. In the engineering practice, BER is measured by collecting statistics over a large number of initially identical pulses. Since different pulses experience different realizations of the noise, averaging over many pulses is actually equivalent to over-the-noise averaging. Repeating the measurement of $B$ many times (each separated from the previous one by a time interval larger than the characteristic time of the disorder variations), one constructs the $\operatorname{PDF} \mathcal{S}(B)$ of $B$. The PDF achieves its maximum at $B_{0}$ that plays the role of typical value of $B$. Even though average distortion of a pulse caused by the noise and disorder is weak, rare but violent events may substantially affect the optical system performance. The probability of such rare events is determined by $\mathcal{S}(B)$ taken at $B \gg B_{0}$.

The PDF of the signal intensity is defined by

$$
P(I \mid\{\hat{m}\})=\left\langle\delta\left(I-\int \mathrm{d} t G(t)|\Psi(Z, t)|^{2}\right)\right\rangle_{\xi},
$$

where averaging is performed over the amplifier noise. Exact calculation of $P(I \mid\{\hat{m}\})$, that accounts for detailed evolution of the signal shape, parametrized by the set of slow modes and radiation parameters introduced in eq. (4), is fairly complicated. However, it can be significantly simplified by noting that for $1 \ll Z \ll D_{\xi}^{-1 / 3}$ the dominant contribution to BER in eq. (6) originates from the slow mode, $y$, in eq. (4) that corresponds to the soliton position shift. Stated differently, in the leading approximation all other slow modes in eq. (4) along with the radiation components $v_{1,2}$ can be neglected and the pulse intensity in eq. (5) can be evaluated by following the stochastic dynamics of the soliton position only. Following the technique developed by Kaup [16], and described in, e.g., [15, 17], one derives

$$
\begin{aligned}
& \partial_{z} y \approx 2 \beta+h_{3}+\mathrm{O}\left(h^{2}\right), \\
& \partial_{z} \beta \approx \int_{-\infty}^{\infty} \mathrm{d} t \frac{\operatorname{Im}\left[e^{-i z} \boldsymbol{\xi}^{(1)}(z, t)\right] \tanh (t)}{\cosh (t)}+\mathrm{O}\left(h^{2}\right),
\end{aligned}
$$

where $\operatorname{Im}\left[e^{-i z} \boldsymbol{\xi}^{(1)}(z, t)\right]$ stands for the imaginary part of $e^{-i z} \boldsymbol{\xi}^{(1)}(z, t)$. The overall soliton shift is a sum of two contributions, $y=y_{\xi}+H_{3}$, where $H_{3} \equiv \int_{0}^{Z} h_{3}(z) \mathrm{d} z$, and $y_{\xi}$ corresponds to the solution of eqs. (8), (9) for $h_{3}(z) \equiv 0$. Substituting the result into eqs. (4), (5) with $v_{1,2}=0$ and $\eta=1$, followed by averaging over $\boldsymbol{\xi}$ in eq. (7), which is naturally translated into averaging over $y_{\xi}$, one arrives at

$$
\begin{aligned}
P(I \mid\{\hat{m}\}) & \approx \int_{-\infty}^{\infty} \frac{3 \mathrm{~d} y_{\xi}}{\sqrt{8 \pi D_{\xi} Z^{3}}} \exp \left[-\frac{9 y_{\xi}^{2}}{8 D_{\xi} Z^{3}}\right] \times \\
& \times \delta\left(I-I_{j}\left(y_{\xi}+H_{3}\right)\right), \quad I_{j}(y) \equiv \int \mathrm{d} t \frac{G(t)}{\cosh ^{2}(t-y)} .
\end{aligned}
$$

Note that the Gaussian statistics of the $y_{\xi}$ component of the soliton position shift (jitter) is due to the so-called Elgin-Gordon-Haus effect $[15,18,19]$. The single-parametric approximation used in deriving eq. (10) will be justified later in the letter. 
Starting with eq. (6), and also making use of the weak-noise assumption $D_{\xi} Z^{3} \ll 1$ that, according to eq. (9), can be interpreted as the statement that typical soliton jitter due to amplifier noise is weak, we derive $B\{\hat{m}\} \approx P\left(I_{\mathrm{d}} \mid\{\hat{m}\}\right)$. This implies that BER can be computed using eq. (10) with $I$ replaced by $I_{\mathrm{d}}$. The $\delta$-function reduces the resulting expression to the sum of contributions, each corresponding to a root of the equation $I_{j}\left(y_{\mathrm{d}}\right)=I_{\mathrm{d}}$. It is clear that the equation has actually two roots, each corresponding to the pulse shifting to the right and left borders of the information slot. For the sake of simplicity and clarity of presentation, we assume that the filter $G$ is $t$-symmetric, in this case the two roots of the aforementioned equation are $\pm y_{\mathrm{d}}$, with the convention $y_{\mathrm{d}}>0$. Then the BER adopts the following form:

$$
\begin{aligned}
B\{\hat{m}\} & \approx B_{0} \cosh \left[\frac{9 y_{\mathrm{d}} H_{3}+\mathrm{O}\left(H_{3}^{2}\right)}{4 D_{\xi} Z^{3}}\right], \\
B_{0} & =\frac{3}{\left|I^{\prime}\left(y_{\mathrm{d}}\right)\right| \sqrt{2 \pi D_{\xi} Z^{3}}} \exp \left[-\frac{9 y_{\mathrm{d}}^{2}}{8 D_{\xi} Z^{3}}\right],
\end{aligned}
$$

where the "cosh" in eq. (11) is constructed out of the two exponential contributions corresponding to the two directions along which the pulse can leave the slot. $H_{3}$ in eq. (11) is an integral of $h_{3}$ that, according to eq. (3), makes it also a zero-mean Gaussian random variable. The tail of the PDF of BER $\mathcal{S}(B)$ is calculated by first expressing $H_{3}$ in terms of $B$ using eq. (11), and then substituting the result into the Gaussian probability measure for $H_{3}$ that follows from eq. (3). This results in

$$
\mathcal{S}(B) \propto \exp \left[-\frac{8 D_{\xi}^{2} Z^{5}}{81 D_{m} y_{\mathrm{d}}^{2}} \ln ^{2}\left[B / B_{0}\right]\right],
$$

valid in the asymptotic region $B \gg B_{0}$. Equation (13) implies that the PDF $S(B)$ has an extended tail. A similar observation has been made already for the linear transmission case [5]; however, it should be emphasized that the form of the extended tail in the nonlinear case is parametrically different. Indeed, the $Z$-dependence of the factor in the exponent in eq. (13) is $\sim D_{\xi}^{2} Z^{5} / D_{m}$, while in the linear case the dependence is $\sim D_{\xi}^{2} Z / D_{m}$. The major difference originates from very different mechanisms of the signal loss in the linear and nonlinear cases: soliton jitter is a single major damaging factor in the nonlinear case, whereas in the linear case the damage is spread among a large number of more or less equally important modes.

We are now in a position to clarify the assumptions leading to eq. (13). First of all, the assumption $Z \gg 1$ means that while passing the distance $Z$ the soliton acquires a large number of the $2 \pi$-long phase turnovers. $D_{\xi} Z^{3} \ll 1$ means that the typical Elgin-Gordon-Haus jitter is small, so that the typical change of the soliton amplitude is even weaker than the change of the position, and the rare event of a pulse loss is primarily due to the soliton shift as a whole. Fluctuations of birefringence that lead to the long PDF tail (13) are small compared to rare but large fluctuations of the noise that produce the error; however, they are not small compared to typical fluctuations of the noise. Stated in formal terms: $H \equiv\left|\int_{0}^{Z} \boldsymbol{h}(z) \mathrm{d} z\right| \gg D_{\xi} Z^{3}$, while $H \ll y_{\xi} \approx y_{\mathrm{d}}=\mathrm{O}(1)$. The contribution to the pulse intensity that is $\mathrm{O}(H)$ originates solely from eq. (8), i.e., there are no other $\mathrm{O}(H)$ contributions coming from any other adiabatic variables (except from $y$ ) and radiation. Strictly speaking, radiation contributes in the $\sim H$ order, however, this contribution contains an additional $1 / Z$ factor making it unimportant. Also note that eq. (13) has been derived under the assumption $\operatorname{tr}[\hat{m}(z)]=0$. The only effect of the $\operatorname{tr}[\hat{m}(z)]$ fluctuations is an additional shift in the soliton position, i.e., it is completely accounted for through a simple renormalization of the $D_{m}$ factor in eq. (13). 
We conclude the letter with a brief discussion of a simple strategy that guarantees an essential reduction of the large fluctuations of BER, described by eq. (13). The major contribution to eq. (13) comes from an $\boldsymbol{h}$-dependent jitter of the soliton position. Thus, making a specially tailored $\boldsymbol{h}(z)$-dependent temporal shift, $t \rightarrow t-t_{\mathrm{cl}}\{\boldsymbol{h}\}$, immediately prior to detection should substantially reduce the outage. In the optical system jargon the timing shift is usually referred to as "setting the clock" compensation. Here we do not discuss the details of such compensation, simply assuming availability of an ideal device capable of performing the complete compensation for the birefringence-induced jitter. Thus, a modified version of eq. (11) that accounts for the "setting the clock" compensation reads $\ln \left[B(\{\hat{m}\}) / B_{0}\right]=\mathrm{O}\left(H^{2}\right) /\left[D_{\xi} Z^{3}\right]$, where the leading second order in $H$ contribution could emerge from corrections to the pulse intensity due to the changes in the soliton parameters other than position shift and phase velocity, as well as the direct contribution due to the radiation emitted by the soliton. A detailed calculation shows that the leading $\mathrm{O}\left(\boldsymbol{H}^{2}\right)$ correction comes from the change in the soliton amplitude, $\eta$, with the other $\mathrm{O}\left(H^{2}\right)$ contributions being sub-leading in $1 / Z$. Moreover, as has been shown in [14], the correction to the soliton amplitude due to PMD is self-averaged at $Z \gg 1: 1-\eta=2 D_{m} Z / 3+\delta \eta$. Since $\delta \eta \propto H^{2} / Z$, one gets from eq. (3) that the PDF of $\delta \eta$ is $\propto \exp \left[-\delta \eta / D_{m}\right]$. (See also [17] for detailed discussion of similar evaluation of the soliton amplitude degradation caused by disorder in the second-order dispersion coefficient.) Therefore, the major effect of the change in the soliton amplitude is a shift of the average BER, $B_{0} \rightarrow \tilde{B}_{0}$, due to the deterministic, $2 D_{m} Z / 3$, part of the amplitude change. The shifted average $\mathrm{BER}$ is given by

$$
\tilde{B}_{0} \simeq B_{0} \exp \left[-\frac{3 y_{\mathrm{d}} A\left(y_{\mathrm{d}}\right) D_{m}}{4\left|I_{j}^{\prime}\left(y_{\mathrm{d}}\right)\right| D_{\xi} Z^{2}}\right],
$$

where

$$
A\left(y_{\mathrm{d}}\right)=-2 \int \mathrm{d} t \frac{G\left(t+y_{\mathrm{d}}\right)[t \tanh t-1]}{\cosh ^{2} t} .
$$

Note thats, when $D_{\xi} Z^{2} \ll D_{m}$, the correction to the typical BER given by eq. (14) is substantial. Describing the PDF of BER at the even larger $B, B \gg \tilde{B}_{0}$, involves accounting for fluctuations of BER due to $\delta \eta$. It is straightforward to check that all other corrections to BER are suppressed by positive powers of $1 / Z$ and/or $D_{\xi} Z^{2} / D_{m}$ small factors. By a similar calculation to the one used in deriving eq. (13), one obtains

$$
-\ln \mathcal{S}(B) \propto D_{\xi} Z^{3} \ln \left[\tilde{B}_{0} / B\right] / D_{m},
$$

which shows that the PDF of BER tail is algebraic in the "setting the clock" compensation case.

We are grateful to V. LEBEDEV for valuable discussions and comments, and to I. GABITOV for useful remarks. We also wish to acknowledge support of Laboratory Director Research and Development Exploratory Research program at Los Alamos National Laboratory. IK acknowledges a personal grant of the Russian Foundation for Promotion of Science, and grant 03-02-16147a of the Russian Foundation for Basic Research. 


\section{REFERENCES}

[1] Agrawal G. P., Nonlinear Fiber Optics (Academic Press, San Diego) 1995.

[2] Hasegawa A. and Kodama Y., Solitons in Optical Communications (Clarendon Press, Oxford) 1995.

[3] Poole C. D. and Nagel J. A., in Optical Fiber Telecommunications, edited by Kaminow I. P. and Koch T. L., Vol. IIIA (Academic Press, San Diego) 1997, p. 114.

[4] Mézard M., Parisi G. and Virasoro M. A., Spin Glass Theory and Beyond (World Scientific) 1987.

[5] Chernyak V., Chertkov M., Kolokolov I. and Lebedev V., to be published in Phys. Rev. E; Opt. Lett., 28 (2003) 2159; Opt. Express, 11 (2003) 1607; JETP Lett., 78 (2003) 198.

[6] Ulrich R. and Simon A., Appl. Opt., 18 (1979) 2241.

[7] Kaminow I. P., IEEE J. Quantum Electr., QE-17 (1981) 15.

[8] Wai P. K. A., Menyuk C. R. and Chen H. H., Opt. Lett., 16 (1991) 1231; Wai P. K. A. and Menyak C. R., Opt. Lett., 19 (1994) 1517; Wai P. K., Kath W. L., Menyuk C. R. and Zhang J. W., J. Opt. Soc. Am. B, 14 (1997) 2967.

[9] Kolokolov I. and Turitsyn S. K., Notes on equation of nonlinear pulse evolution in randomly birefringent optical fiber, submitted to JETP (2003).

[10] Desurvire E., Erbium-Doped Fiber Amplifiers (John Wiley \& Sons) 1994.

[11] Feller W., An Introduction to Probability Theory and Its Applications (Wiley, New York) 1957.

[12] Manakov S. V., Sov. Phys. JeTP, 65 (1973) 505.

[13] Lakoba T. I. and Kaup D. J., Phys. Rev. E, 56 (1997) 6147.

[14] Chertkov M., Gabitov I., Kolokolov I. and Lebedev V., JeTP Lett., 74 (2001) 535.

[15] Falkovich G., Kolokolov I., Lebedev V. and Turitsyn S. K., Phys. Rev. E, 63 (2001) 025601

[16] Kaup D. J., Phys. Rev. A, 42 (1990) 5689; 44 (1991) 4582.

[17] Chertkov M., Chung Y., Dyachenko A., Gabitov I., Kolokolov I. and Lebedev V., Phys. Rev. E, 67 (2003) 036615.

[18] Elgin J. N., Phys. Lett. A, 110 (1985) 441.

[19] Gordon J. P. and Haus H. A., Opt. Lett., 11 (1986) 665. 